Kommt der O. amygdaloides var. $\beta$. ambigua Cogn. Monogr. p. 1052 am näehsten.

45. O. congestiflora (Naud.) Oogn.

Sao Paulo.

\title{
Planktonstudien über den Wörther-See in Kärnten.
}

Von Dr. Karl v. Keißler (Wien).

Nachdem ich vor einiger Zeit Gelegenheit gehabt hatte, von den größeren Kärntner Seen den Millstätter- und Ossiacher-See planktologisch zu untersuchen, ging ich im Jahre 1905 daran, auch einige Beobachtungen über das Plankton des Wörther-Sees zu sammeln ${ }^{1}$ ). Dieselben beziehen sich auf den Zeitraum von März bis September, u. zw.: einzelne Fänge Ende März und anfangs April, eine größere Anzahl von Fängen von Mitte Juni bis Ende Juli, einzelne Fänge in der zweiten Hälfte August und anfangs September. Die Mehrzahl der Planktonzüge wurden zwischen Pörtschach und Töschling, einige auch am westlichen Ende des Sees bei Velden und am östlichen bei Seekirn ausgeführt, doch ergaben diese keinerlei Unterschiede gegenüber den Fängen bei Pörtschach und Töschling.

Zunächst möchte ich eine Liste der für den genannten Zeitraum konstatierten Planktonten geben, um späterhin verschiedene allgemeine Betrachtungen anzustellen.

Übersicht der Planktonten.

(Zeitraum Ende März bis Aufang September 1905, mit Ausschluß des Monates Mai.)

Peridineae.

Ceratium hirundinella 0 , F. M.

März, April: fehlend; Juni: selten; Juli-September: mäßig häufig.

Die Exemplare sind breit, dreihörnig (mit gerade vorgestrecktem seitlichen Horn), haben eine Größe von $115-135$ II 55-60 $\mu$, entsprechen also derjenigen Form, die Zederbauer ${ }^{2}$ ) als $C$. carinthiacum beschreibt und auf Tab. $\nabla$, fig. 2, für den Wörther-See abbildet. Innerhalb des Zeitraumes von Juni bis September zeigen sich an Ceratium keine auffallenden Veränderungen in der Gestalt.

1) Einige Angaben über diesen See finden sich schon in Breh m und Zederbauer, Beiträge zur Planktonuntersuchung alpiner Seen III (Verhandl. d. k. k. zool.-bot. Ges. Wien, Jahrg. 1905, p. 235).

2) Vgl. dessen Abhandlung "Ceratium hirundinella in den österreichischen Alpenseen “ (Österr. botan. Zeitschr. 1904, p. 127); ferner Brehm und Zederbauer, 1. c. p. 240. 
Peridinium cinctum Ehrbg.

März, April: fehlend; Juni-September: sehr selten.

\section{Flagellatae.}

Dinobryon divergens Imh.

März: mäßig hä ufig; April : häufig; Juni-_Juli : fehle nd; August: sehr: selten; September: selten.

Dinobryon stipitatum Stein.

März: fehlend; April: sehrselten; Juni-Juli : fehlend; August-September: sehr selten. var. lacustre Chod.

März, April; Juni-Juli: fehlend; August-September: sehr selten.

\section{Bacillariaceae.}

Fragilaria crotonensis Kitt.

März: sehr selten; April: hänfig; Juni-Juli: sehr selten; August, September: selten.

Die Exemplare haben eine Breite von ea. 95-100 $\mu$, entsprechen also der var. $\gamma$. subprolongata Schröt. u. Vogl. in Vierteljahrsschr. d. Naturf. Ges. Zürich XLVI (1901), p. 196, fig. $2 a, B$. Merkwürdigerweise zeigen sich in dem ganzen zur Beobachtung gelangten Zeitraum keinerlei Variationen in betreff der Breite der Bänder, wie dies sonst mehrfach beobachtet wurde und worüber ja auch Schröter und $\nabla$ ogler in der oben zitierten Abhandlung berichten. Auf den Bändern findet sich häufig ein Parasit.

Fragilaria virescens Ralfs.

März--September: ganz vereinzelt.

Synedra ulna Ehrbg. var. splendens Brun.

März, April: fehlend; Juni-Juli; mäßig bäufig; August, September: fehlend.

Frusteln nicht geknöpft, $300 \mu$ und darüber lang.

Asterionella formosa Hassk. var. gracillima Grun. et var. subtilis Grun.

März: fehlend; April: mäßig häufig; Juni: erst sehr selten, dann mäßig häufig: Juli: erst mäßighäufig, dann häufig; August, September: sehr selten.

Sterne meist 6--8 strahlig, mit ca. $150 \mu$ Durchmesser.

Tabellaria fenestrata Kuetz.

März, April: sehr selten.

Cyclotella comta Kuetz.

März, April: fehlend; Juni-Juli: häufig; August, September: mälig häufig. 


\section{Schizophyceen.}

Chroococcus minutus Naeg.

April-September: ganz vereinzelt.

Microcystis spec.

Juni-September: ganz vereinzelt.

Clathrocystis aeruginosa Henfr. selten.

April-Juli : fehlend; August: mä ßig häufig; September :

Lyngbya limnetica Lemm. in Botan. Centralbl. Bd. 76 (1898), p. 154.

April-Juli : fehlend; August, September: mäßig häufig.

Die Spezies dürfte, soweit sich bei der Zartheit der Fäden $(0 \cdot 5 \mu$ Durchmesser) feststellen läßt, richtig bestimmt sein.

\section{Chlorophyceae.}

Cosmarium bioculatum Bréb.

März, April: fehlend; Juni-September: sehr selten.

Sphaerocystis Schröteri Chod.

März: fehlend; April: șehr selten; Juni: mäßig häufig; Juli-September: selten.

Tritt in zahlreichen Entwicklungsstadien aut.

Botryococeus Braunii Kuetz.

März: fehlend; April: sehr selten; Juni : erst selten, dann mäßig häufig; Juli-September: selten.

Grüne und rötliche Kolonien in annähernd gleichem Verhältnis gemengt. Es finden sich auch einige auffallend gestaltete Kolonien, die besondere Entwicklungsstadien darzustellen scheinen, ähnlich jenen, die ich seinerzeit für den Wolfgang-See beschrieben ${ }^{1}$ ) und auf Tab. I abgebildet habe.

Es fanden sich namentlich die auf Fig. $5 \mathrm{za}$ sehenden Formen. Die vegetative Vermehrung von Botryococcus Braunii Kuetz. scheint nach diesen Beobachtungen sich so zu vollziehen, daß die Gallerte sich lockert, daß zwisehen den mit Zellen versehenen Partien derselben sich Gallertfäden bilden, die immer länger werden, bis wir eine bäumchenartige Kolonie vor uns haben, von der sich endlich die mit Zellen versehenen rundlichen Partien lostrennen, welche anscheinend den Ausgangspunkt für neue Botryococcus-Kolonien bilden.

Scenedesmus spec.

März, April: fehlend; Juni-September: sehr selten.

1) Vgl. Verhandl. d. zool.-bot. Gesellsch. Bd. 52 (1902), p. 307. 
Dictyosphaerium Ehrenbergianum Naeg.

März, April ; Juni-Juli: fehlend; August: selten; September: mäßig häufig.

Oocystis solitaria Wittr.

März, April: fehlend; Juni-September: selten.

Raphidium Brauni Naeg. var. lacustre Chod. in Bull. de l'herb. Boiss. Tome V (1897), p. 291.

März-Juli : fehlend; August, September: selten.

Richteriella botryoides Lemm.

März-Juli: fehlend; Angust--September: sehr selten. Als Verunreinigung spärlich Koniferenpollen vorkommend ${ }^{\mathbf{1}}$ ).

Wenn ich nunmehr das Plankton des Wörther-See im allgemeinen zu charakterisieren suche, so muls bemerkt werden, daß während der Monate März bis September (1905) das Phytoplankton gegenüber dem Zooplankton entschieden überwiegt. Die Hauptrepräsentanten des Planktons während der einzelnen Monate sind folgende Organismen:

März (2. Hälfte) :

April (1. Hälfte):

Mai ?

Juni (Mitte):

Juli (Mitte) :

August (2. Hälfte): Lyngbya limnetica, ferner Cyclotella, endlich Clathrocystis.

September (1. Hälfte): Lyngbya limnetica, ferner Cyclotella, endlich Dictyosphaerium.

Über die Art des Auftretens der einzelnen Planktonten im Wörther-See während des von mir beobachteten Zeitraumes gibt die folgende Tabelle Aufschlub:

1) Bezüglich der Zusammensetzung des Zooplanktons wäre folgende annähernde Angabe zu machen: Protozoa. Difflugia spec. März, April: fehlend; Juni: sehr selten; Juli: mäßig häufig; August, September: selten. - Rotatoria. Anuraea cochlearis Gosse, Notholca longispina Kell., Chromogaster spee. März, April: fehlend; Juni-September: se hr selten. Polyarthra platyptera Huds. März, April, Juni: fehlend; Juli-September: sehr selten; Asplanchna spec. August, September: sehr selten. - Crustaceae. Cyclops spee. März: fehlend; April: sehr selten; Juni: selten; Juli, August: sehr selten; September: selten. Diaptomus spec. März, April: sehr selten; Juni-Juli: selten; Angust: sehr selten; September: selten. Bosmina spec. April: sehr selten. Daphnia spec. Juni-September: sehr selten. Leptodora hyalina Leyd. Juni, Juli : einzelne Stüeke. Nauplien. März: sehr selten; April: mäßig häufig; Juni-August : sehr selten; September: selten. 
Wörther-See (1905).

\begin{tabular}{|c|c|c|c|c|c|c|c|c|}
\hline Planktonten & 总产 & 营营 & 虫志 & 毠当 & 墨: & 㿣: & 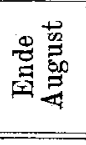 & 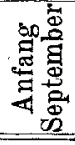 \\
\hline Dinobryon (di- & & & & & & & & \\
\hline Ceratium ......... & 0 & $0_{0}^{10}$ & s & $\begin{array}{l}0 \\
\mathrm{~s}\end{array}$ & $\mathrm{mh}$ & $\begin{array}{c}0 \\
\mathrm{mh}\end{array}$ & $\mathrm{mh}$ & $\mathrm{mh}$ \\
\hline Cyctotella (comta). & 0 & 0 & $\mathbf{h}$ & sh & $\mathrm{h}$ & $\mathrm{h}$ & $\mathrm{mh}$ & $\mathrm{mh}$ \\
\hline Asterionella....... & 0 & $\mathrm{mb}$ & ss & $\mathrm{mh}$ & $\mathrm{mh}$ & $\mathbf{h}$ & ss & ss \\
\hline $\begin{array}{l}\text { Synedra }: . . . . . . \\
\text { Fragilaria }\end{array}$ & 0 & 0 & $\mathrm{mh}$ & $\mathbf{h}$ & $\mathrm{mh}$ & 0 & 0 & 0 \\
\hline nensis) .......... & SS & h & Ss & ss & ss & $\mathbf{s}$ & $\mathbf{s}$ & $\mathbf{s}$ \\
\hline Raphidium $\ldots \ldots$. & 0 & 0 & 0 & 0 & 0 & 0 & $\mathrm{~s}$ & $\mathrm{~s}$ \\
\hline Botryococcus..... & 0 & ss & ss & $\mathrm{mb}$ & $\mathbf{s}$ & $\mathrm{s}$ & $\mathrm{s}$ & s \\
\hline Dictyosphaerium.. & 0 & 0 & 0 & 0 & 0 & ss & $\mathbf{s}$ & $\mathrm{mh}$ \\
\hline Sphaterocystis ..... & 0 & ss & $\mathrm{mh}$ & s & $s$ & $s$ & $\mathrm{~s}$ & $\mathbf{s}$ \\
\hline Clathrocystis...... & 0 & 0 & 0 & 0 & 0 & 0 & $\mathrm{mh}$ & $\mathbf{s}$ \\
\hline Lyngbya......... & 0 & 0 & 0 . & 0 & 0 & 0 & $\mathrm{mh}$ & $\mathrm{mh}$ \\
\hline Difflugia ...... & 0 & 0 & ss & sS & $\mathrm{mh}$ & s & $\mathfrak{s}$ & $\$$ \\
\hline Diaptomus ...... & ss & ss & $\mathbf{s}$ & $\mathbf{s}$ & ss & ss & SS & $\mathrm{s}$ \\
\hline Nauplius-Stadien .. & ss & $\mathrm{xuh}$ & sS & ss & Ss & ss & ss & $\mathbf{s}$ \\
\hline
\end{tabular}

Nach der eben im allgemeinen skizzierten Zusammensetzung des Planiktons des Wörther-Sees erfahren wir vor allem, daß das in anderen Seen oft so reichlich auftretende Ceratium hier, ähnlich wie im Ossiacher-See ${ }^{1}$, eine geringe Rolle zu spielen scheint. Bemerkenswert ist ferner das an Menge nicht unbedeutende Auftreten von Lyngbya limnetica Lemm. im Monat August und September, ein Phytoplanktont, der, soviel mir bekannt, bisher in keinem österreichischen Alpensee nachgewiesen warde, sowie das Vorkommen von Clathrocystis, eine Alge, die in den österreichischen Alpenseen im Gegensatz zu den norddeutsehen Seen nicht häufig zu sein scheint und die ich selber nur in einigen kleineren Kärntner Seen, wie im Worstnigg- und Jeserzer-See bei Velden, ferner im Plaschischen-See bei Klagenfurt gefunden habe ${ }^{2}$ ). Hervorzuheben ist endlich das Auftreten von Raphidium und Richteriella.

In Rücksicht auf die verschiedenen Jahreszeiten finden wir im März und April im Wörther-See in Übereinstimmung mit einer Anzahl anderer österreichischer Alpenseen ein Dinobryon-

1) Vgl. Keis ler, Mitteilungen über das Plankton des Ossiacher-Sees in Kärnten (Osterr. botan. Zeitschr. Jahrgang 1905, p. 101).

2) Vgl. Ke io ler, Beitrag zur Kenntnis des Planktons einiger kleinerer Seen in Kärnten (Ibidem, Jahrg. 1906, p. 53). Möglicherweise ist auch das von mir für den Brenn-See bei Villach angegebene Vorkommen von Coelosphaerium in ein Vorkommen von Clathrocystis abzuändern, da ja bekanntlich jugendliche Stadien von Clathrocystis den Kolonien von Coelosphaerium sehr ähnlich sehen. (Vgl. Österr. botan. Zeitschr. Jahrg. 1904, p. 58.) 
Plankton, im Juni und Juli, ähnlich wie im Ossiacher-, Millstätter- und bis zu einem gewissen Grade im Hallstätter-See, ein Diatomaceen-, speziell ein Cyclotella-Plankton, im August und September endlich ein Gemisch von einem Lyngbya- (Chroococcaceen-) Plankton und einem Cyclotella-Plankton.

Die wechselnde Zahl der pflanzlichen Planktonten ersehen wir aus folgendem:

$\begin{array}{lcccccc} & \text { März } & \text { April } & \text { Juni } & \text { Juli } & \text { August } & \text { September } \\ \text { Phytoplankton } & 6 & 9 & 14 & 14 & 21 & 20 \text { Arten, }\end{array}$
demnach ein Maximum an Arten im August und September.

Wenn ich nunmehr die Zusammensetzung des Planktons des Wörther-Sees zu verschiedenen Jahreszeiten mit derjenigen anderer größerer Kärntner Seen, wie des Ossiacher- und Millstätter-Sees, vergleiche, so weit mir eben Beobachtungen zur Verfügung stehen, dann zeigt sich, daß auf der einen Seite zwischen dem Plankton dieser drei Seen gewisse Ähnlichkeiten bestehen, daß aber andererseits auch die Unterschiede nicht unbeträchtliche sind. Einige Tabellen mögen dies vielleicht am besten illustrieren. Ich gebe zunächst eine Tabelle, in welcher das Juli-Plankton des Ossiacher-, Wörther- und Millstätter-Sees einander gegenübergestellt sind. Gemeinsam ist allen drei Seen das spärliche Vorhandensein von Dinobryon und Fragilaria crotonensis, ferner die Häufig keit von Cyclotella comta (u. zw. im Wörther-See die typische C. comta, in den beiden anderen die var. melosiroides Kirchn.).

\begin{tabular}{|c|c|c|c|}
\hline Planktonten & $\begin{array}{c}\text { Ossiacher-See } \\
\text { Juli } 1904\end{array}$ & $\begin{array}{c}\text { Wörther-See } \\
\text { Juli } 1905\end{array}$ & $\begin{array}{c}\text { Millstätter-See } \\
\text { Juli } 1903\end{array}$ \\
\hline Ceratium........... & $\mathrm{s}$ & $\mathrm{mh}$ & sh \\
\hline Dinobryon ......... & ss & ss & 0 \\
\hline Fragilaria crotonensis & ss & $\mathbf{s}$ & ss \\
\hline Cyclotella........... & sh & h & sh \\
\hline Asterionella ......... & 0 & h & ss \\
\hline Synedra............ & $\mathbf{m h}$ & ss & 0 \\
\hline Melosira............ & $\mathbf{m h}$ & 0 & 0 \\
\hline Dictyosphaerium..... & $\mathbf{m h}$ & ss & 0 \\
\hline Botryococeus........ & 0 & $\mathrm{~s}$ & $\mathbf{m h}$ \\
\hline
\end{tabular}

Doch finden wir auch einige Unterschiede. Der OssiacherSee weist z. B. ein Vorkommen von Melosira als nicht unwesentlichen B e standteil des Juli-Planktons auf, während im Wör therund Millstätter-See diese Diatomee fehlt; desgleichen enthält der Ossiacher-See in ziemlicher Menge Synedra und Dictyosphaerium, welche beide im Juli im Wörther-See eine untergeordnete Rolle spielen, im Millstätter-See im Juli ganz fehlen. Der Wörther-See hinwiederum führt im Juli-Plankton in grö ß erer Menge Asterionella, im Millstätter-See dagegen ist diese Diatomacee im Juli sehr selten, im Ossiacher-Seofehlt sie 
ganz. Im Millstätter-See endlich ist im Juli Botryococcus ziemlich häufig, während diese Alge im Wörther-See nicht sehr hervortritt und im Ossiacher-See im Juli ganz fehlt. In betreff der Häufigkeit des Vorkommens von Ceratium im Juli verhalten sich, wie die obige Tabelle lehrt, die drei Seen auch versehieden.

Nicht uninteressant ist es vielleicht, auch das Septemberplankton des Wörther- und Millstätter-Sees einer vergleichenden Betrachtung zu unterziehen, zu welchem Behufe ich zunächst eine diesbezügliche Tabelle vorausschicke:

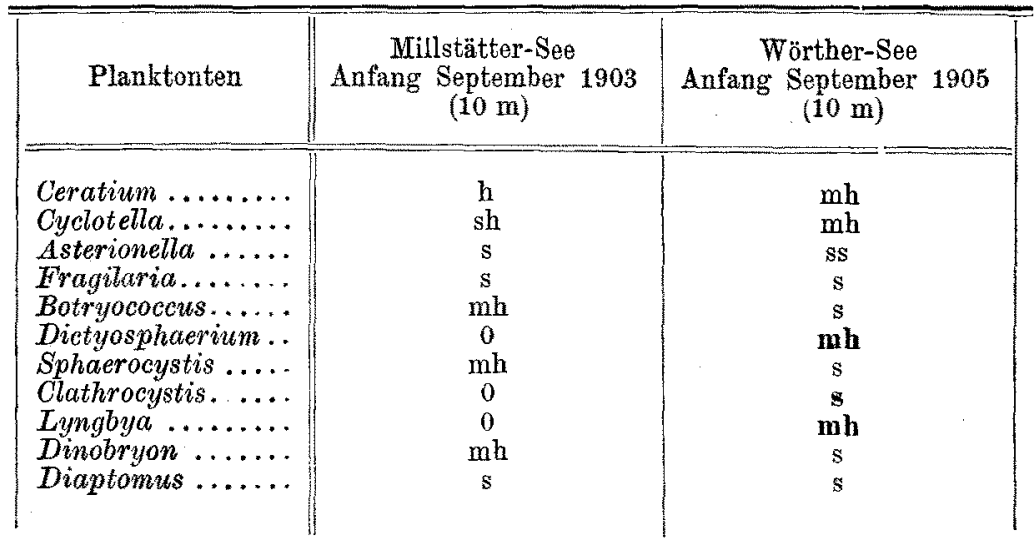

Wir sehen aus der obigen Zusammenstellung, daß zwischen dem Septemberplankton der genannten zwei großen Kärntner Seen, von dem spärlichen Auftreten von Asterionella und Fragilaria abgesehen, ziemliche Unterschiede bestehen. Diese äußern sich erstens darin, da 1 diverse Planktonten in beiden Seen in an. gleicher Menge vorkommen, wie Ceratium, Cyclotella, Dinobryon etc., zweitens darin, daß im Septemberplankton des WörtherSees Planktonten (zum Teil sogar als ma isgebende Faktoren) auftreten, die dem Mills tätter-See fehlen, so Lyngbya, Dictyosphaerium und Clathrocystis.

Endlich möchte ich noch in Kürze das Plankton des WörtherSees mit demjenigen zweier kleinerer, dem Wörther-See benachbarter Wasserbecken, des Faaker- und Klopeiner-Sees, vergleichen, soweit mir hierüber Beobachtungen zur Verfügung stehen ").

Wörther-See: Cyclotella comta, ferner Synedra, Hauptvertreter Faaker-See: $\left.\begin{array}{l}\text { Cydlich Sphaerocystis } \\ \text { Cyclla comta, ferner Ceratium }\end{array}\right\} \quad \begin{aligned} & \text { im Juni- } \\ & \text { Plankton. }\end{aligned}$

Gemeinsam ist also dem Wörther- und Faaker-See die Häufigkeit von Cyclotella im Juni-Plankton, ähnlich wie in

I) Siehe Keißler, Beitrag zur Kenntnis des Planktons einiger kleinerer Seen in Kärnten (Österr. botan. Zeitschr. Jahrgang 1906, p. 53). 
einigen anderen österreichischen Alpenseen im Sommer Cyclotella eine wichtige Rolle spielt. Im uibrigen herrscht aber zwischen dem Wörther- und Faaker-See keine Ubereinstimmung.

Wörther-See: Cyclotella comta, ferner Asterionella und Synedra, endlich Ceratium

Klopeiner-See: Ceratium, ferner Dinobryon, end- $\begin{gathered}\text { Mitte Juli. } \\ \text { lieh Peridinium }\end{gathered}$

Zwischen dem Wörther-See und Klopeiner-See finden wir in Rücksicht auf die Zusammensetzung des Juli-Plankton keine $\ddot{A} \mathrm{hn}$ lichkeit. Der einzige, beiden Seen gemeinsame Hauptvertreter ist Ceratium, das im Klopeiner-See jedoch an die erste Stelle, im Wörther-See nur an die vierte Stelle rangiert. Cyclotella comta fehlt dem Juli-Plankton des Klopeiner-See.

\section{Über einige Mißbildungen an Blüten der Gattung Pedicularis.}

Von Josef Stadlmann, stud. phil.

(Aus dem botanischen Institute der $\mathrm{k} . \mathbf{k}$. Universität in Wien.)

(Mit Tafel IV.)

In Steiningers verdienstvoller Arbeit "Beschreibung der europäischen Arten des Genus Pedicularis" findet sich Seite 17 (Sep. Abdr.) bei Pedicularis Barrelieri folgende Bemerkung, mit der ich bei meinen Untersuchungen einigermaßen in Widerspruch kam. Er schreibt: "Ped. Barrelieri neigt sebr zur Pelorienbildung, indem nicht gerade selten Exemplare angetroffen werden, welche neben normalen zygomorphen Bläten durch Fehlschlagen der Oberlippe scheinbar aktinomorphe Blüten besitzen, ans deren Kronenrôhre ganz normal entwickelte Staubfäden lang herausragen und der Blüte dadurch ein recht sonderbares Aussehen verleihen. Auber bei $P$. Barrelieri hatte ich nur Gelegenheit, Plorienbildung bei der $P$. rostrata $L$. und bei $P$. tuberosa L. zu bemerken."

Penzig ${ }^{1}$ ) hat Steiningers Angaben in sein Handbuch übernommen.

Von einer Pelorienbildung konnte man aber nach der allgemeinen Definition dieser Erscheinung ${ }^{2}$ ) doch nur dann sprechen, wenn die umgestaltete Blüte aus einer zygomorphen zu einer aktinomorphen wird. Als klassisches Beispiel dafür ist die bekannte Pelorie von Linaria vulgaris Mill. zu nennen. Steininger ge-

1) O. Penzig, Pflanzenteratologie II., p. 216, 217. p. 449 .

2) Vgl. auch C. K. Schneider, Mlustr. Handwörterbuch der Botanik, 